\title{
EFFECTS OF DIGITAL BLOCK ON PULSE OXIMETRY IN SHOCK SIMULATION
}

Hassanali Soltani MD, Jalal Hashemi MD, Kamran Tavakol MD

Department of Anaesthesia and Critical Care, Isfahan University of Medical Science, Isfahan, Iran, 8174673461

INTRODUCTION: Pulse oximetry is impaired by hypotension and peripheral vasoconstriction (1). Digital block may cause increase in tissue perfusion and hence improving the parameters of pulse oximetry (2). The purpose of this study was to investigate the effect of digital block on $\mathrm{spaO}_{2}$, lag time and height of plethysmographic wave (PW) of pulse oximeter, in presence of shock simulation in upper extremity.

METHODES: After approval of the proposal by institutional ethics committee and obtaining the informed consent, 34 patients under general anesthesia and elective surgery were selected. In each patient, one hand randomly underwent simulated shock by cooling $\left(<35^{\circ} \mathrm{c}\right)$, elevation of hand $(40 \mathrm{~cm})$ and inflation of arm cuff $(30 \mathrm{mmHg})$. As the kinetic energy in artery is dissipated in climbing a vertical hydrostatic gradient, the absolute pressure in artery decreasing by $0.75 \mathrm{mmHg}$ per centimeter vertical distance up to the heart. Depending on the level of elevation of trial hand $(40 \mathrm{~cm})$ and inflation of arm cuff $(30 \mathrm{mmHg})$, the hydrostatic pressure in distal port of this hand decreases approximately $60 \mathrm{mmHg}$.

Lag time, height of PW (in millimeter) and $\mathrm{spaO}_{2}$ were measured in middle and small fingers of this hand and compared to that of middle finger of another hand. Shocked middle finger was blocked by lidocaine $2 \%$ and above parameters were measured in 15 th and 20th minutes after digital block and compared to small shocked finger and middle finger of non-shocked hand. Statistical analysis was performed with ANOVA.

RESULTS: Mean height of PW in shocked and blocked, shocked non- blocked and control fingers are shown in table (Mean \pm SD). There were no significant differences between three fingers in lag time and $\mathrm{spaO}_{2}$.

\begin{tabular}{|l|l|l|l|}
\cline { 2 - 4 } \multicolumn{1}{c|}{} & \multicolumn{3}{c|}{ Height of PW $(\mathrm{mm})$} \\
\hline Finger & $\begin{array}{l}\text { Before } \\
\text { block }\end{array}$ & 15 th min & 20 th min \\
\hline Shocked \& blocked & $11.5 \pm 4.2$ & $16.2 \pm 6^{? *}$ & $21 \pm 5.9^{? *}$ \\
\hline Shocked \& non-blocked & $10.8 \pm 4.3$ & $11.4 \pm 4.3$ & $11.8 \pm 4.3$ \\
\hline Control & $11.3 \pm 4.4$ & $10.7 \pm 4.3$ & $11.2 \pm 3.9$ \\
\hline \multicolumn{3}{|c|}{ P $<0.05$ vs shocked and non- blocked finger } \\
$*$ \\
P $<0.05$ vs control finger
\end{tabular}

DISCUSSION: This study showed that digital block may improve height of pletysmographic wave in shocked condition and better pulse oximetric signal detection.

\section{REFERENCES:}

1) Indian J. Anaesth. 46: 261-268

2) Anesth Analg. 73: 815-817 